prostatic hypertrophy and not its cause. We bclieve that this legitimate question can be answered in the negative. We have endeavored to show in the above examinations that the increase in size of the glands was either immediately or secondarily due to the direct inflammatory exudates or to the reaction on the part of the tissues to these processes, i. c., interstitial hyperplasia.

Granting that our observations in this series of specimens is correct, the origin of the inflammation is yet to be ascertained. In certain of our cases $(30,52,55$, and 56) the sections apparently indicated that the primary inflammatory process had originated about the posterior urethra. Had all our sections been chosen with the determination of this point in view, it is probable that a larger percentage would have shown this condition. But at best. such deductions from the histologic picture are uncertain and more or less speculative. In our opinion clinical evidence is much more trustworthy. It will hardly be questioned that prostatitis most frequently results from, or follows, posterior urethritis, and posterior urethritis is usually primarily or secondarily of gonococcic origin. Asserting as we do, however, that prostatic hypertrophy is the direct result of chronic prostatitis, by the same chain of clinical evidence we are forced to the conclusion that it follows posterior urethritis from whatever cause. For the reasons stated above chronic proliferative prostatitis is more frequent in the aged than in the young.

From our study of prostatic hypertrophy we believe that the usual story of this disease is as follows: Given a posterior urethritis, infection of the ducts and alveoli follows. This is accompanied by inflammatory infiltration about the walls of the infected sac. Following this inflammatory exudate (Fig. 1), which may involve large portions of the gland, fibroblasts and plasma cells appear and acute interstitial hyperplasia becomes inaugurated, as shown in Figs. 2 and 3 . The changes which now follow depend largely on what portion of the gland is involved. If the hyperplasia be most marked in the peripheral portions, the acini become compressed and the epithelium atrophies (Fig. 4) and from such acini small fibromata may result-Fig. 5. If the process be more slow and infiltration still active, the condition shown in Fig. 6 may follow. Should the fibrosis be most marked about the ducts or the glands, constriction of these tubes may produce pictures like that shown in Fig. 7, which may be followed by that shown in Fig. 9. Now, if fibrosis be marked about the acini the condition shown in Fig. 11 may happen; on the other hand, acute inflammation with pus formation may take place, as seen in Fig. 10. If proliferation of the alveolar epithelium be excited, pictures like that of Fig. 8 are produced and if this irritation result in malignant cell proliferation true cancer may form (Fig. 12) finally with carcinomatous infiltration of the interstitiumFig. 13.

This work on prostatic hypertrophy has purposely been founded on histologic findings only, for the reason that in the early days, when the study of prostatic hypertrophy was necessarily purely clinical, or aided only by gross observations or rude microscopic methods, the general impression was that the disease was inflammatory in nature. This fact has already been stated in our introduction. This idea held until, after incomplete sporadic microscopic studies, a theory was formed, founded purely on the histology of the disease, that prostatic hypertrophy was a true tumor formation and idiopathic in its origin. Nearly all text-books on greneral. pathology now give this view and the greatest of pathologists have favored the theory either by copying what others have written or by confining their personal observations to a few specimens only. Serious histologic studies of the hypertrophic prostate bave not been numerous and for this reason, if for no other, we are much indebted to the recent, most careful and thoroughly scientific study of Ciencanowski, who has fully demonstrated by his histologic observations that hypertrophy of the prostate is an inflammatory disease.

This study was taken up by one of us with the itlea of disproving the statements of Ciencanowski and satisfying himself of the truth of the idea expressed in nearly all works on pathological anatomy. The result has been a complete conversion to the views of Ciencanowski; whose conclusions we endorse fully, as far as our work has covered his field. We therefore take no credit to ourselves for originality of thought or discovery, leaving all credit to the clinical observers who first advanced the theory and to Ciencanowski who first fully and finally proved its truth.

We may bricfly state our conclusions as follows:

1. Prostatic hypertrophy of the aged is the result of chronic prostatitis.

2. It most frequently arises from chronic posterior urethritis, of whatever cause.

3. True neoplasms of the prostate are rare and are not concerned in the production of prostatic hypertrophy.

4. Carcinoma is apt to occur in the hypertrophied prostate as a result of the chronic inflammatory process.

\section{SARCOMA OF THE UTERUS.}

\section{S. FAIRCHILD, M.D.}

Professor of Surgery, Medical Department Drake University : Surgeon to St. Joseph's Hospital.

CLINTON, IOWA.

During the present year lady 65 years of age came under my care presenting the following history and symptoms. The mother of several children, she had enjoyed good health until about two years ago, when she began to be troubled with constipation and pain in the lower part of abdomen and a sense of weight and pressure in the pelvis. These symptoms gradually increased. About six months prior to coming under my observation the symptoms became more acute, when she had, according to her statement, a discharge of fluid from the bowels. 'This gave her a measure of relief, but soon the same chronic condition she had previously suffered from appeared and continued on with some increase in severity until she came under my care in August of last year. A physical examination revealed the uterus to be uniformly enlarged to two or three times its normal size and appeared to be firmly impacted in the pelvis. There was no discharge from the organ and the symptoms seemed to be largely due to pressure. The general condition of the patient appeared to be good, although she had lost in weight and was somewhat anemic. This could be accounted for in part at least, on the ground of constipation and impaired digestion.

The age of the patient and the fact of the growth appearing after the menopause suggested malignancy, but the absence of positive symptoms of cancer indicated the possibility of a non-malignant growth. Assuming the probability of an enlarged and myomatous uterus I opened the abdomen. The peritoneum was very much thickened and there was found a considerable quantity of free fluid in the abdominal cavity. The abdominal 
wound was made sufficiently large to admit of a free examination of the uterus after the intestines were walled back by large abdominal pads.

The bladder was normal in appearance. The uterus was uniformly enlarged and occupied the usual position in the pelvis and reached upwards to a point corresponding to a uterus enlarged to the fourth month of pregnancy. The anterior surface of the uterus presented a grayish-white appearance, and fluctuated on palpation. The sigmoid was well crowded over to the left. The uterus was firmly adherent on its posterior surface. Masses of new growth extended out on either side to the ovaries and broad ligaments. It was at once apparent that we had to deal with a sarcomatous uterus and that any attempt to remove it would involve a dangerous hemorrhage with small prospect of getting all the diseased tissue. I could not see my way clear to secure safe hemostasis on either side of the uterus, and having in mind the certain and speedy return of the disease in such cases, I determined not to expose the life of the patient in the useless attempt to remove the organ. I opened the cyst on the anterior surface of the uterus just sufficient to explore the interior, about one ounce of clear fluid escaped, revealing a very red and irregular velvety surface. The thick cyst wall became flaccid when the fluid escaped, which erabled me to surround the threequarter inch incision with a purse-string suture slightly inverting the edges, thus closing the opening completely. I now reinforced the purse string with a few additional sutures. Catgut was used in both instances. I folded over the sutured surface a strip of iodoform gauze, which I allowed to extend out of the lower angle of the wound. inserting a secondary suture to be tied when the gauze was removed on the sixth or eighth day; otherwise the wound was carefully closed. On the eighth day the gauze was removed, followed by the discharge of some serous fluid and the secondary suture tightened. The patient was discharged and returned home, a distance of 150 miles, in good condition at the end of the second week.

It was my impression that the less we disturbed the diseased organ the better it would be for the patient. I doubted the wisdom of opening the cyst, but the unusual appearance of the organ and the desire to discover the condition inside the cyst led me to do so.

My personal experience with diffuse sarcoma of the uterus is extremely limited, but the literature of the subject does not give much warrant for operative procedures when the disease has advanced so far as to make the diagnosis easy before an operation is undertaken. In early cases where scrapings have revealed the sarcomatous nature of the disease better results may be expected, or in somewhat later cases, when the disease is clearly confined to the organ and the tying off can be easily accomplished without leaving any part. an operation may be warranted. But when the disease has plainly spread out over the broad ligaments, even to a moderate extent, no good can be accomplished.

A recent examination shows that the growth has rapidly increased since the exploratory operation and her general health has correspondingly declined and the fatal termination is not far off. I realize the fact that a diagnosis as to the character of a morbid growth without a microscopic study lacks in scientific accuracy, but the clinical importance of arriving at a conclusion at the operating table was, in my judgment, of the utmost importance. If the disease was malignant I ought to have removed it; if non-malignant, I am very sure I would have shortened her life. I recalled the special features in the history of the case, that a few months previous to the operation a quantity of fluid had escaped from her bowels, giving her for a time some relief. This was no doubt due to the emptying of a cyst lying in contact with the sigmoid. I took into account the general appearance of the mass, the free fluid in the abdominal cavity, the adhesions and the extension of the growth on to broad ligaments. I had never before seen a cyst sarcoma of the uterus or a sarcoma which had undergone cystic degeneration. But I was so firmly impressed with the idea that I had one before me that 1 had no hesitation in acting on the theory that it would be much better for the patient to let it alone. What the antecedents of the growth were I do not know. But it is quite probable that a fibro-myoma involving the fundus had existed for perhaps a long time which had more recently undergone cystic and sarcomatous degeneration. An important diagnostic symptom was absent, namely, a uterine discharge; but it may be assumed that the endometrium and sub-mucous tissues were not involved. However, there was no distinct tumor and the organ appeared to be uniformly enlarged, a condition which would seem to favor the probability of such a general involvement as to lead to hemorrhages. It may be observed that recently a moderate loss of blood has occurred on one or two occasions. Since the operation, and for some time before, she has suffered severe pain in the abdomen, has lost much in flesh and has become very anemic.

The development of a diffuse sarcoma of the uterus after the menopause is much less frequent than carcinoma, but that it does occur late in life is well established. and it has particularly been observed to occur as the result of degenerative changes in fibro-myomas, which have no doubt remained stationary for a long time. When these growths, which appear to give no serious trouble and make no advancement, begin to take on a new growth attended with pain, loss of weight and anemia they may be looked upon with great suspicion if the change occurs after the menopause. This fact suggests the danger which may arise in allowing these apparently harmless uterine tumors to remain, and whether the risk of letting them alone is not greater than the risk of life in their removal. There can be no question on this point, if after 50 years of age a more or less rapid growth is discovered, but then it may be too late for the best results. Is it not true that any form of uterine growth is a menace to life at some time. even if it is apparently stationary?

\section{THE RELATIONSHIP OF ANTISTREPTOCOC- CUS SERUM TO THE TREATMENT OF PUERPERAL SEPSIS.}

\section{GEORGE E. RANNEY, M.D.}

Ex-President Michigan State Medical Society; President United States lension Lxamining Board at Lansing; State Inspector of Communicable Diseases: Member of the Michigan State Board of Registration in Medicine. Etc. LANSING, MICH.

Since the first disturbances of unknown origin in the puerperal state, up to the time of Semmelweiss, who stimulated investigation of fever in the puerperal state, this subject has been the bone of contention and the modus operandi for its successful treatment has been the highest aim of all clinicians.

Serum therapy has been deemed ideal for the treatment of diseases due to specific organisms. Pasteur, Koch, Behring and others contended that the bodily 